\title{
Chapter 5 \\ Experimental Investigation on Nonlinear \\ Dynamic Evolution Patterns of Cracks \\ in Rock Failure Process
}

\subsection{Introduction}

Based on CT scanning results of sandstone, CT images were analyzed using the MATLAB software and the evolution pattern of mesoscopic cracks were explored under uniaxial cyclic load/unload. Fractal dimension was used to define the degree of chaos in the process of cracks propagation. System entropy model was established using the fractal dimension. Verifying the relationship between the areas and lengths of cracks and stress, a crack growth factor model was proposed. It revealed the nonlinear dynamic evolution patterns of cracks propagation in the process of sandstone failure using CT parameters. This model provided a new method for predicitng rock dynamic hazard. Compared with the actual test results using the three-dimensional reconstruction and numerical simulation of roof and bottom sandstone's mesostructure of initial stage, the correctness of numerical simulation was verified.

The rock failure process does not occur in a short time, it is a significant accumulation process, which is determined by the brittle characteristics of rock. In the process of cyclic load/unload; the higher concentrated stress will appear at the tip of rock cracks, which will lead to the initiation and expansion of cracks inside the rock, and gradually through the new macroscopic cracks leading to rock failure.

Most scholars studied the cracks evolution using the similar model test, and the process of cracks initiation, expansion and penetration failure could be observed intuitively and clearly. Nolen-Hoeksema and Gordon (1987) conducted a compression test on the marble with prefabricated sloping fissures. Through the observation of cracks tip and the failure mode, it showed that the propagation process of cracks was asymmetric, and the damage inside the rock could be predicted using the observation of cracks distribution on the specimen's surface. Many scholars studied the evolution pattern of cracks on the two-dimensional plane using a microscope, and the data information of microstructure of rock was obtained. Wong (1982), and Wong et al. (2004) obtained that the microstructure of granite 
crack was changed. The rapid development of CT provided a variety of simple and effective methods for statistical analysis of data. Priest and Hudson (1976, 1979) acquired the geometrical characteristics of fractured rockmass. Reyes and Einstein (1991) observed the evolution of gypsum specimen under uniaxial static loading, the failure mechanism of fractured body was controlled using the geometric distribution of initial prefabricated cracks defects. Shen et al. (1995), Shen and Barton (1997) proposed new failure criteria, and improved the Griffith criterion, by which the test results could be explained reasonably. Bobet (2000) carried out the gypsum model test with the distribution of cracks defects under uniaxial loading, and the initiation, expansion and penetration mechanism of overlapping and non-overlapping distributions were obtained. Hoxha et al. (2005) concluded that the extended form of secondary cracks was significantly affected by the change in prefabricated cracks location.

In this chapter, CT scanning technology and digital image processing technology were used to obtain the cracks image under different load levels. The nonlinear dynamic model of cracks propagation evolution was revealed, and the nonlinear dynamic model of cracks propagation was established. At present, the evolution patterns of cracks propagation still stays in the whole process of cracks initiation-extension-failure of microscopic level, and does not explain the macroscopic failure pattern of rock through the process of cracks propagation. a three-dimensional visualization model of cracks was established, and a crack growth factor model was proposed, which provided a new way to study the transition from microscopic scale to macroscopic scale.

\subsection{Pattern of Nonlinear Dynamics Evolution of Rock Cracks}

It is necessary to study the microscopic cracks changes of rocks in order to explain the macroscopic mechanical properties of geomaterials. The complexity and heterogeneity of rock cracks and the nonlinear characteristics of its high degree of disordered distribution was constituted in rock failure process (Lin et al. 2000; Wong et al. 2001). Complex materials were previously difficult to quantitatively describe by fractal or chaotic methods. In this chapter, the fractal dimension of cracks in the two-dimensional plane was obtained, and the fractal dimension was used to analyze the sandstone cracks. The entropy model of rock system was established based on the fractal dimension, the mesoscopic mechanics of cracks was reveals from initiation and propagation. The cracks growth factor model was established using the deduction of cracks chaos discrimination, this model can reveal the expansion and evolution of sandstone cracks. 


\subsubsection{Fractal Theory}

The concept of fractal was first proposed by American mathematician B.B. Mandelbort. The Latin "Fractus" was the earliest source of fractal, and all irregular movements could be described by fractal in Mandelbrot's theory (Mandelbrot and Pignoni 1983; Mandelbrot 1977). Fractal geometry is a rapid new development discipline in recent years; it can describe any disorder movement. Fractional dimension is an important concept of fractal geometry, and its value can be a fraction. Fractal dimension became fractal geometry with the continuous development of society. It was pointed out that fractal dimension was a powerful tool for studying the patterns of most physics, and the treatment of those very irregular shapes must rely on the theory of fractal geometry (Goldberger et al. 2002; Faloutsos and Gaede 1996). The irregular, rough profile of rock fracture surface could be seen as self-similarity. Heping $\mathrm{X}$ et al. applied new geometric-fractal geometry to describe this irregularity. Fractal model of intergranular fracture was established, and the composite fracture between particles and intergranular was obtained. Fractal characteristics of ruptured rock surface were acquired using electron scanning and optical fractal analysis. Finally, the relationship between fractal dimension and macroscopic mechanical parameters of rock fracture was obtained (Xie and Chen. 1988, Heping X. 1989).

Fractal geometry mainly has two parts: the linear fractal and the nonlinear fractal (Mandelbrot 1999). The linear fractal theory uses some irregular curves with self-similar as the research object, and the fractal dimension is the continuous view of its basic view. The nonlinear fractal theory takes the geometries under the nonuniform linear transformation groups and the geometric shapes under the nonlinear transformation groups as the object of their properties. In the nonlinear fractal theory, the fractal dimension of nonuniform linear transformation reflects the essential characteristics of richness and complexity of nature. In this chapter, using the fractal dimension in the process of change under uniaxial cyclic load/unload, the evolution of cracks was also seemingly chaotic, it is necessary to quantitatively describe the expansion and evolution of cracks for realizing the disorder cracks propagation.

\subsubsection{Results Analysis on Fractal Dimension of Rock Cracks}

\subsubsection{Calculated Procedure of Fractal Box Dimension}

With the application and development of fractal geometry theory, the definition and measurement methods of fractal dimension are more abundant. There is no uniform definition of fractal dimension; the fractal dimension and the choice of measurement 
methods are discriminative for different research objects. There are six fractal dimensions, such as perimeter-area relation, power law spectrum, self-affine fractal method, box counting method, modified yardstick method. Based on the definition of box dimension, the fractal dimension of box dimension was used to calculate the fractal dimension in the process of rock cracks. Box-dimension method ( $\mathrm{Li}$ et al. 2009; Foroutan-pour et al. 1999) was also called Box method, network coverage, or coverage method. Using a square or a radius of epsilon (called a box) to cover the surface or curve of an object, and the number of boxes could alter as the epsilon changes. If the measured objects have fractal properties, the following relationships are satisfied:

$$
\mathrm{N}(\varepsilon)=a \varepsilon^{-D}
$$

where $a$ is a constant. Taking the logarithm of both sides, it can be described as follow:

$$
\mathrm{D}=\lim _{\varepsilon \rightarrow 0} \frac{\ln (N(\varepsilon) / a)}{\ln (1 / \varepsilon)}
$$

a set of data $\mathrm{N}(\varepsilon)-\varepsilon$ could be gotten by covering, the double logarithmic graph could be drawn, the scale-free region on the $\ln \varepsilon-\ln N$ graph could be identified. Using the least square method, the slope was obtained, and the absolute value of slope was the fractal dimension value.

The specific steps of cracks fractal dimension were calculated using the box counting method.

1. For rock cracks image of a pixel-sized $M \times M$, it was found as a three-dimensional surface. The length and width are $M$, the height is $L$, using the pixel series of image to represent, it is set to $L=256$.

2. The cracks plane image $(M \times M)$ was divided into a grid of size, and the coordinate scale of "height" was divided $R \times R$ equally, the unit of division was $R \times L / M$. Therefore, the three-dimensional curved surface was divided into many three-dimensional "boxes". The key of this division method had the same division "number" of width and height direction.

3. Within each the grid was divided into the size of $R \times R$, the pixel value $u$ and pixel minimum value $b$ was founded. The minimum to the maximum pixel was calculated, pixels how many boxes to cover, box number to remember $n(i, j)$, assuming that now the grid was $(i, j)$. That was $n(i, j)=[(u-b+R-1) / R]$, in the equation for the whole symbol.

4. Take the sum of each box $R \times R$, Counted as $N$, that was $N=\operatorname{sum}(n(i, j))$.

5. In theory, the fractal dimension at this time was $D=-\log N / \log R, R$ was close to infinity. $R$ was limited in reality. Therefore, the sizes of $R$ value were change, a group $N$ was found, and then using the linear fitting method, it showed that the slope of straight line was the required fractal dimension $D$. 


\subsubsection{Results Analysis on Fractal Dimension of Sandstone Cracks}

With good self-similarity of surface cracks in the sandstone, the cracks distribution in the sandstone was handled by the box counting method of fractal geometry using the two-dimensional CT scanning algorithm. The calculation results were shown in Tables 5.1, 5.2 and Figs. 5.1, 5.2.

As shown in Figs. 5.1 and 5.2, fractal dimension curve showed as follow:

1. In the initial unloaded status, the roof and bottom sandstone samples were scanned, and the fractal dimensions of layers were different. Therefore, the initial damage of sandstone was not uniform, and it was an initial damage medium mainly based on the existence of microcracks.

2. As shown in Figs. 5.1 and 5.2, the evolution of fractal dimension in this process was divided into three stages. The first stage was the unloading stage - the ends of the first stage of the cycle load/unload. With the stress increases, the cracks begin to expand, the complexity of cracks structure and the fractal dimension increases. The second stage was the end of the first stage of cycle and unloading to the end of the second stage of cyclic load/unload, fractal dimension of different layers was almost the same. However, fractal dimension of the 650 layer slice was almost unchanged because the initiation of cracks was continued to meet at this stage, the overall shape and structure of cracks were from the complex to simple, and the fractal dimension stabilization was occured. At the other level, due to the presence of new cracks around the through cracks, the complexity of cracks structure and the fractal dimension increased. The third stage was the end of the second stage of cycle load/unload to the specimen

Table 5.1 Calculated results of fractal dimensions of roof sandstone

\begin{tabular}{l|l|l}
\hline Scan phase & Scan sections & Fractal dimensions \\
\hline \multirow{2}{*}{ Before loading $(0 \mathrm{MPa})$} & 350 layer & 1.8769 \\
\cline { 2 - 3 } & 450 layer & 1.8767 \\
\cline { 2 - 3 } & 550 layer & 1.8767 \\
\cline { 2 - 3 } After the first phase $(9 \mathrm{MPa})$ & 650 layer & 1.8768 \\
\hline \multirow{2}{*}{ After the second phase $(27 \mathrm{MPa})$} & 350 layer & 1.8789 \\
\hline & 450 layer & 1.8784 \\
\cline { 2 - 3 } & 550 layer & 1.8787 \\
\cline { 2 - 3 } & 650 layer & 1.8792 \\
\hline \multirow{2}{*}{ After failure $(81.9 \mathrm{MPa})$} & 350 layer & 1.8792 \\
\cline { 2 - 3 } & 450 layer & 1.8787 \\
\cline { 2 - 3 } & 550 layer & 1.8789 \\
\cline { 2 - 3 } & 650 layer & 1.8796 \\
\hline & 350 layer & 1.8764 \\
\hline & 450 layer & 1.8765 \\
\cline { 2 - 3 } & 550 layer & 1.8765 \\
\hline & 650 layer & 1.8766 \\
\hline
\end{tabular}


Table 5.2 Calculated results of fractal dimensions of bottom sandstone

\begin{tabular}{|c|c|c|}
\hline Scan phase & Scan sections & Fractal dimensions \\
\hline \multirow[t]{4}{*}{ Before loading (0 MPa) } & 350 layer & 1.8759 \\
\hline & 450 layer & 1.8757 \\
\hline & 550 layer & 1.8760 \\
\hline & 650 layer & 1.8758 \\
\hline \multirow[t]{4}{*}{ After the first phase (6 MPa) } & 350 layer & 1.8767 \\
\hline & 450 layer & 1.8765 \\
\hline & 550 layer & 1.8767 \\
\hline & 650 layer & 1.8769 \\
\hline \multirow[t]{4}{*}{ After the second phase (18 MPa) } & 350 layer & 1.8769 \\
\hline & 450 layer & 1.8769 \\
\hline & 550 layer & 1.8771 \\
\hline & 650 layer & 1.8770 \\
\hline \multirow[t]{4}{*}{ After failure (37.5 MPa) } & 350 layer & 1.8754 \\
\hline & 450 layer & 1.8755 \\
\hline & 550 layer & 1.8756 \\
\hline & 650 layer & 1.8755 \\
\hline
\end{tabular}

Fig. 5.1 The tendency of fractal dimensions at each layer of roof sandstone on different stress conditions

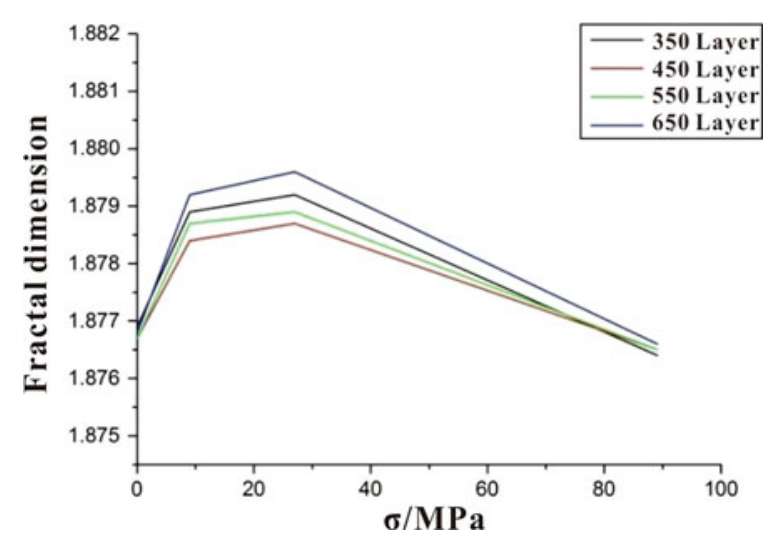

destruction. At this stage, the cracks were intermittent to accelerate, and many macroscopic main cracks appeared. The failure surface was formed in the crack-dense area, and the specimens were cracked along the failure surface. However, under the stress, the non-main cracks gradually were closed, and the shape and structure of cracks were gradually developed toward the simplification resulting in the fractal dimension decreasing rapidly.

3. Based on the analysis and calculation of fractal dimension of cracks at each scanning level, it was concluded that the propagation and evolution of cracks in the rock failure process, they were quantitatively described by the fractal dimension, that is to say, the damage degree of rock was quantitatively described by the size of fractal dimension. 
Fig. 5.2 The tendency of fractal dimensions at each layer of bottom sandstone at different stress conditions

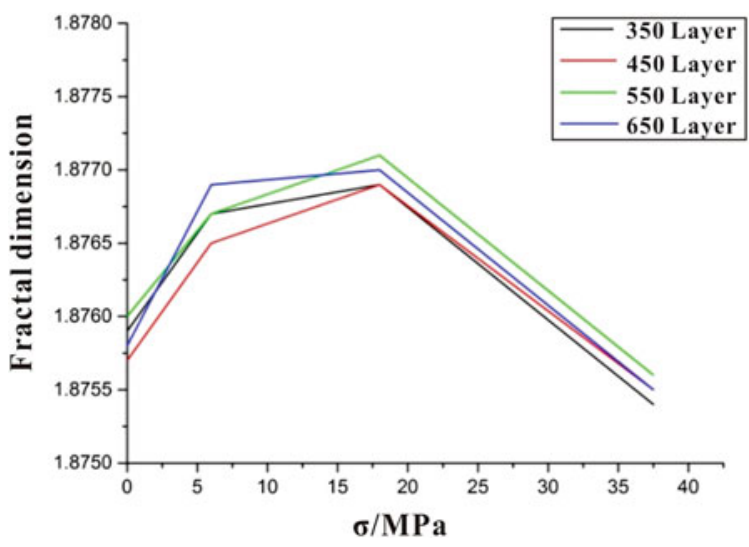

\subsubsection{Entropy Model of Rock System Based on Fractal Dimension}

The entropy can measure the disorder degree and the entropy of a large number of microscopic particles in the system from the microscopic point of view, and the entropy increases with the disorder degree and the chaotic status of the granular matter in the system. The chaos of system is proportional to the entropy of system. This relationship is first established by Boltzmann, called the Boltzmann theorem, as follow:

$$
S=k \times \ln W
$$

where $S$ is the entropy, the description of system mechanical status; $k$ is the Boltzmann constant; $W$ is the chaos of system.

Therefore, if the chaos degree of rock system is defined, the entropy of rock system $S$ can be revealed, and the meso-structure evolution of rock failure process can be verified from disorder to order.

The more the microscopic state of system, the greater the degree of confusion. The system could be quantified with the number of micro-state quantitative description. Since the fractal dimension can express the complexity of system (i.e., the degree of chaos), the fractal dimension of sandstone cracks was calculated under cyclic load/ unload. The microcracks began to expand and gradually converged into cracks, this extension and the process of cracks distribution showed a trend of reducing the dimension of system from the disorder to the orderly dynamic characteristics. Therefore, the fractal dimension was used to define the degree of confusion $W$.

The Gauss Amp function expression is:

$$
y=y_{0}+A \mathrm{e}^{-\frac{\left(x-x_{c}\right)^{2}}{2 \omega^{2}}}
$$


where $y_{0}$ is the offset of $y$-axis direction of curve, and $A$ is the difference between the maximum and minimum values of curve, that is the amplitude of function. The $A \mathrm{e}^{-\frac{\left(x-x_{c}\right)^{2}}{2 \omega^{2}}}$ can be defined as a normal distribution of deformation, the standard normal distribution formula: $f(x)=\frac{1}{\sqrt{2 \pi} \sigma} \mathrm{e}^{-\frac{(x-\mu)^{2}}{2 \sigma^{2}}}$, the symmetry axis is $x_{c}, \omega$ for the relevant parameters to meet $2 \omega=F W H M / \operatorname{sqrt}(\ln (4))$, according to the equation to calculate its size.

The function is symmetrical about $x=\mathrm{x}_{c}$, and when $x=\mathrm{x}_{c}$ the extremes are obtained, If $A>0$, the maximum value is obtained at that point; if $A<0$, the minimum value is obtained at that point. The distribution of function is basically similar to the normal distribution, when $A>0$, the opening goes down; $A<0$, the opening is upward. The larger the $\omega$ value, the more the function image is "thin"; the smaller $\omega$, the more function image is "too thick".

As shown in Figs. 5.1 and 5.2, the fractal dimension of each section of roof and floor sandstone was changed and then fitted with the above function. Each parameter in the equation was given practical significance. The dependent variable $y$ is the fractal dimension $D, x_{c}$ is the lower limit stress $\sigma_{\mathrm{c}}$ of the secondary cyclic load/unload, $y_{0}$ is the fractal dimension value $D_{0}$ when the stress is $\sigma_{0}, A$ is the fluctuation range of fractal dimension value, and $\omega$ is the relevant parameter. Figures 5.3 and 5.4 showed the fitting parameters. Table 5.3 showed the relevant parameters.

As shown in Table 5.3, the fractal dimension of top and bottom sandstone was in accordance with the distribution of Gauss Amp function in the process of cracks propagation. Hence, the evolution formula of and stone crack propagation based on fractal dimension was obtained as follows:

$$
D=D_{0}+A \mathrm{e}^{-\frac{\left(\sigma-\sigma_{\mathrm{c}}\right)}{2 \times \omega}}
$$

Fig. 5.3 Fitting curves of fractal dimensions and stress curves of roof cracks

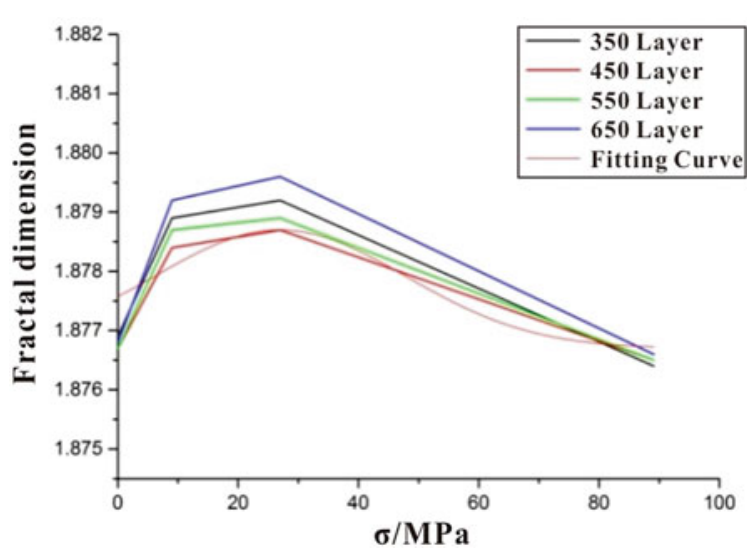


Fig. 5.4 Fitting curves of fractal dimensions and stress curves of floor cracks

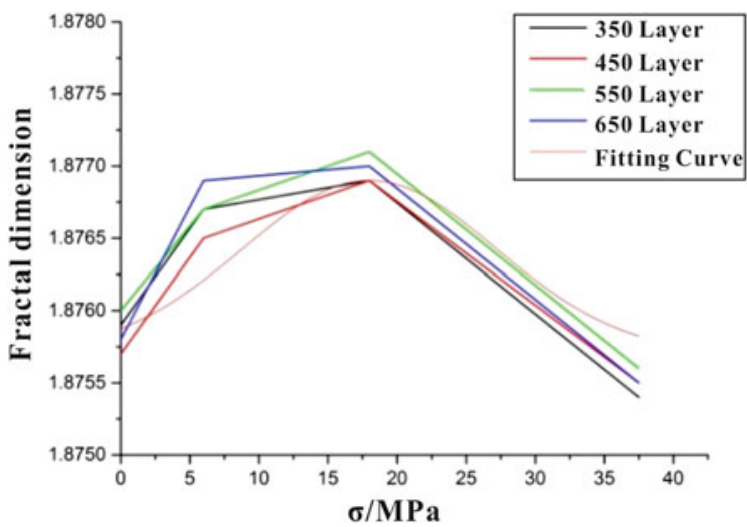

Table 5.3 Parameters and equations for fitting curves

\begin{tabular}{l|l|l|l|l|l}
\hline \multirow{2}{*}{ Rock type } & \multicolumn{4}{|l|}{ Related parameters } & \multirow{2}{*}{ Equations for fitting curves } \\
\cline { 2 - 5 } & $\mathrm{D}_{0}$ & $\mathrm{~A}$ & $\sigma_{\mathrm{c}}$ & $\omega$ & \\
\hline Roof sandstone & 1.8767 & 0.002 & 27 & 20.97 & $D=1.8767+0.002 \mathrm{e}^{-\frac{(\sigma-27)^{2}}{2 \times 20.97}}$ \\
\hline Floor sandstone & 1.8759 & 0.003 & 18 & 8.72 & $D=1.8759+0.003 \mathrm{e}^{-\frac{(\sigma-18)^{2}}{2 \times 8.72}}$ \\
\hline
\end{tabular}

where the system chaos $W$ in Eq. (5.4) can be expressed by the fractal dimension of cracks. Therefore, it can put the formula (5.6) into the formula (5.4), the sandstone system entropy model is:

$$
S=\mathrm{k} \times \ln \left(D_{0}+A \mathrm{e}^{-\frac{\left(\sigma-\sigma_{\mathrm{c}}\right)}{2 \times \omega}}\right)
$$

where $k$ is a constant; $\sigma_{\mathrm{c}}$ is the lower limit stress value of the secondary cyclic load/ unload; $\sigma$ is the stress value at any time; $D_{0}$ is the fractal dimension value of initial state; $\omega$ is the coefficient in the Gauss Amp function; The range of volatility; $S$ is the system entropy of sandstone failure process.

This model quantitatively was described the evolution of system entropy in the process of sandstone cracks propagation and evolution, which provided a theoretical basis for the rock fracture. This experiment was only carried out CT scanning test on the top and bottom sandstone rock samples without other rocks test, but other rock samples may meet the model. Thus, the model could be extended to other hard rock rocks: $S=\mathrm{k} \times \ln \left(D_{0}+A \mathrm{e}^{-\frac{\left(\sigma-\sigma_{\mathrm{C}}\right)}{2 \times \omega}}\right)$, where the value of $\omega$ is related to the lithology of rock.

In this chapter, the entropy model of rock system combined the change of fractal dimension and the change of system entropy in the process of rock failure, the evolution of system entropy under the action of uniaxial cyclic load/unload was described quantitatively. It was proved that the dimensionality reduction, entropy reduction and order were unified in the process of rock failure. The dynamic 
characteristics of rock system from disorder to order could be described by the process of dimension reduction and entropy reduction. It was shown that the rock specimens were exhibited a critical behavior of destabilization as the stress increases during loading. The entropy of rock specimens and the fractal dimension of cracks were reduced, the distribution of cracks was ordered and the rock specimen was destabilized. The model described the evolution of rocks from microstructure to macroscopic failure.

\subsection{Chaotic Characteristics of Cracks Evolution in Rock Failure Process}

In nonlinear dynamics, the motion status of matter is deterministic and unpredictable, and this phenomenon is called chaos. Chaotic phenomenon is similar to the random motion and unpredictable. However, these research results show that chaos can be determined in the nonlinear dynamics, and the instability of motion status of material in the system leads to its unpredictability. With the continuous development of nonlinear dynamics, chaos is one of the most common motion status in nonlinear dynamics, and it is also the essential attribute of nonlinear dynamical system. In this chapter, the chaotic motion of cracks was derived from the nonlinear variation in the process of cracks propagation, and there was some kind of internal relation of certainty and randomness in the process of cracks change. Therefore, it is necessary to obtain the chaotic characteristics in the process of cracks propagation for predicting rockburst.

\subsubsection{Chaos Theory}

Chaotic motion has a certain non-linear feature, it is gradually converged to a certain point in the system of disorderly status-chaos, and there is no universally recognized mathematical definition. The chaos is defined as:

(Li-Yorke Chaos definition) Continuous mapping or point mapping $\mathrm{F}:[a, b] \times R \rightarrow[a, b],(\mathrm{x}, \lambda) \rightarrow F(x, \lambda)$ is chaos, If the following conditions are as follow:

1. There are periodic periods of all cycles;

2. There is a countless subset $\mathrm{S} \subset[a, b]$, where $S$ does not contain a periodic point:

(1) If any $x, y \in S$,

$$
\lim _{n \rightarrow \infty} \inf \left|F^{n}(x, \lambda)-F^{n}(y, \lambda)\right|=0
$$


(2) If any $x, y \in S(x \neq y)$,

$$
\lim _{n \rightarrow \infty} \sup \left|F^{n}(x, \lambda)-F^{n}(y, \lambda)\right|>0
$$

(3) If any $x \in S$ with $F$ is any periodic point,

$$
\lim _{n \rightarrow \infty} \sup \left|F^{n}(x, \lambda)-F^{n}(p, \lambda)\right|>0
$$

So far, chaos is the inherent law of seemingly disordered phenomena. Although there is no definite definition of chaos, it has mainly some common characteristics as follows: sensitivity to the initial value, intrinsic randomness, boundedness ergodicity, universality, self-similarity. The local area in the chaotic system appears to be complicated and disordered. However, the system is self-similar, and the self-similar structure is superimposed in the system, which makes the whole system seem to be chaotic and irregular.

\subsubsection{Discrimination of Chaotic Characteristics Defined by Li-Yorke}

This chapter uses the defined Li-Yorke criterion for cracks evolution, chaotic characteristics is derived assuming without any loading condition, the sandstone CT number for initial and with the increasing of stress cracks generated which can cause the new crack energy release. Therefore, the change process of describing the density of sandstone can be used to use trigger-growth-triggered cracks chain growth model. The density of microcrack was assumed, the generalized driving force was proportional to the production of cracks, and the CT number $n_{i+1}$ of sandstone can be expressed as: when the loading went to the stage of $i+1$.

$$
n_{i+1}=F_{1}(\sigma) \cdot n_{i}-F_{2}(\sigma) \cdot\left(N_{0}-n_{i}\right) \cdot X_{i}
$$

where

$N_{0}$ is total CT number of sandstone samples;

$\mathrm{X}_{i}=\frac{n_{i}}{N_{0}}$ dimensionless;

where $F_{1}(\sigma)$ is survival ratio of cracks, the quantitative description of cracks closure effect, no dimensionless scalar;

where $F_{2}(\sigma)$ is driving force depends on the magnitude of stress, the dimensionless scalar. 
According to the testing results of the whole process and the experimental results of literature, it can be assumed that:

$$
F_{2}(\sigma)=\frac{\ln \sigma}{\ln \sigma_{c}}
$$

where $\sigma_{\mathrm{c}}$ is uniaxial compression strength

Take the formula (5.11) into the formula (5.10):

$$
n_{i+1}=F_{1}(\sigma) \cdot n_{i}-\frac{\ln \sigma}{\ln \sigma_{c}} \cdot\left(N_{0}-n_{i}\right) \cdot \frac{n_{i}}{N_{0}}
$$

based on the formula (5.11):

$$
\begin{aligned}
X_{i+1} & =F_{1}(\sigma) \cdot X_{i}-F_{2}(\sigma) \cdot X_{i} \cdot\left(1-X_{i}\right) \\
& =F_{2}(\sigma) \cdot X_{i} \cdot\left[\frac{F_{1}(\sigma)-F_{2}(\sigma)}{F_{2}(\sigma)}+X_{i}\right]
\end{aligned}
$$

command:

$$
\begin{gathered}
\mathrm{x}_{\mathrm{i}}=\frac{\mathrm{F}_{2(\sigma)}}{\mathrm{F}_{1}(\sigma)-\mathrm{F}_{2(\sigma)}} \mathrm{x}_{\mathrm{i}} \\
\mathrm{F}_{2}(\sigma)=\frac{\left[\mathrm{F}_{1}(\sigma)-\mathrm{F}_{2(\sigma)}\right]^{2}}{\mathrm{~F}_{2(\sigma)}}
\end{gathered}
$$

where $F_{2}(\sigma)$ is the single valued function of $F_{1}(\sigma)$ and $F_{2}(\sigma)$, which is defined as the generalized driving force; It's the sum of single valued functions. $X_{i}$ is the single valued functions.

Based on the formulas (5.14) and (5.15), (5.10) and (5.14) can be expressed as:

$$
\mathrm{X}_{\mathrm{i}+1}^{2}=\mathrm{f}\left(\mathrm{F}_{2(\sigma)}, \mathrm{x}_{\mathrm{i}}\right)=\mathrm{F}_{2(\sigma)} \mathrm{x}_{\mathrm{i}}\left(1+\mathrm{x}_{\mathrm{i}}\right)
$$

The standard formula of Logistic:

$$
x_{n+1}=f\left(x_{n}, \mu\right)=\mu x_{n}\left(1+x_{n}\right)
$$

Equation (5.16) is a one-dimensional nonlinear mapping, which is a Logistic equation with chaotic characteristics of Logistic mapping. As shown in Tables 5.4 and 5.5, the theoretical calculation results of CT number of the top floor sandstone specimen are $0-6.27 \%$ and $0-7.66 \%$ respectively. Therefore, the evolution of sandstone cracks in the single axial cyclic loading condition has chaotic characteristics. 
Table 5.4 Theoretical calculation results of roof sandstone specimens are compared with experimental results

\begin{tabular}{|c|c|c|c|c|}
\hline The scanning stage & $\begin{array}{l}\text { Scanning } \\
\text { layer }\end{array}$ & $\begin{array}{l}\text { Experimental results } \\
\text { of CT numbers }\end{array}$ & $\begin{array}{l}\text { Computed results } \\
\text { of CT numbers }\end{array}$ & $\begin{array}{l}\text { Error } \\
(\%)\end{array}$ \\
\hline \multirow{4}{*}{$\begin{array}{l}\text { The stage of } \\
\text { unloading }\end{array}$} & 350 layer & 576.03 & & \\
\hline & 450 layer & 592.74 & & \\
\hline & 550 layer & 589.88 & & \\
\hline & 650 layer & 607.59 & & \\
\hline \multirow{4}{*}{$\begin{array}{l}\text { The end of the first } \\
\text { stage of loading }\end{array}$} & 350 layer & 610.61 & 572.32 & 6.27 \\
\hline & 450 layer & 614.75 & 589.87 & 4.05 \\
\hline & 550 layer & 606.70 & 575.35 & 5.17 \\
\hline & 650 layer & 611.07 & 606.02 & 0.83 \\
\hline \multirow{4}{*}{$\begin{array}{l}\text { The end of the second } \\
\text { stage of loading }\end{array}$} & 350 layer & 622.99 & 600.31 & 3.64 \\
\hline & 450 layer & 626.96 & 607.32 & 3.13 \\
\hline & 550 layer & 624.17 & 604.46 & 3.16 \\
\hline & 650 layer & 627.23 & 615.58 & 1.86 \\
\hline \multirow[t]{4}{*}{ The stage of failure } & 350 layer & 629.86 & 617.96 & 1.89 \\
\hline & 450 layer & 635.19 & 628.71 & 1.02 \\
\hline & 550 layer & 633.27 & 619.75 & 2.13 \\
\hline & 650 layer & 637.96 & 629.67 & 1.30 \\
\hline
\end{tabular}

Table 5.5 Theoretical calculation results of floor sandstone specimens are compared with experimental results

\begin{tabular}{|c|c|c|c|c|}
\hline The scanning stage & $\begin{array}{l}\text { Scanning } \\
\text { layer }\end{array}$ & $\begin{array}{l}\text { Experimental results } \\
\text { of CT Numbers }\end{array}$ & $\begin{array}{l}\text { Computed results } \\
\text { of CT Numbers }\end{array}$ & $\begin{array}{l}\text { Error } \\
(\%)\end{array}$ \\
\hline \multirow{4}{*}{$\begin{array}{l}\text { The stage of } \\
\text { unloading }\end{array}$} & 350 layer & 553.64 & & \\
\hline & 450 layer & 563.59 & & \\
\hline & 550 layer & 573.29 & & \\
\hline & 650 layer & 589.38 & & \\
\hline \multirow{4}{*}{$\begin{array}{l}\text { The end of the first } \\
\text { stage of loading }\end{array}$} & 350 layer & 583.53 & 538.84 & 7.66 \\
\hline & 450 layer & 579.37 & 544.99 & 5.93 \\
\hline & 550 layer & 573.68 & 548.55 & 4.38 \\
\hline & 650 layer & 567.98 & 551.96 & 2.82 \\
\hline \multirow{4}{*}{$\begin{array}{l}\text { The end of the second } \\
\text { stage of loading }\end{array}$} & 350 layer & 598.51 & 562.74 & 5.98 \\
\hline & 450 layer & 596.87 & 563.38 & 5.61 \\
\hline & 550 layer & 593.09 & 571.47 & 3.65 \\
\hline & 650 layer & 590.60 & 581.03 & 1.62 \\
\hline \multirow[t]{4}{*}{ The stage of failure } & 350 layer & 602.74 & 573.05 & 4.93 \\
\hline & 450 layer & 603.83 & 577.38 & 4.38 \\
\hline & 550 layer & 602.13 & 583.03 & 3.17 \\
\hline & 650 layer & 607.13 & 594.11 & 2.14 \\
\hline
\end{tabular}




\subsubsection{Construction of Crack Growth Factor Model}

A function or a curve of Logistic is a common form of $S$ function named after Pierre Francois Velure in 1844 or 1845 in order to reveal the relationship between the curve and population growth. The generalized curve of Logistic can be used to describe the shape of $S$ curve that represents the population growth $(P)$. The population grows exponentially at the initial stage; as the number grows, the population reaches saturation and the growth rate decreases. Finally, the growth rate decreases to zero when the population grows to the maximum allowable environment. As shown in Fig. 5.5, the function is expressed as follow: $\mathrm{y}=A_{2}+\frac{\left(A_{1}-A_{2}\right)}{\left(1+x / x_{0}\right)^{p}}$, where $x$ means any moment, $y$ represents the number of people corresponding to the time $x, A_{1}$ is the population at the initial moment, $A_{2}$ is the largest population that can be accommodated under natural and environmental conditions, $x_{0}$ represents the time corresponding to $A_{2} / 2, p$ is correlation coefficient.

Based on the Sect. 5.2.2, it showed that the chaotic characteristics of sandstone were chaotic under uniaxial cyclic load/unload. In this chapter, the cracks lengths and areas were calculated as the ordinate and the abscissa with the stress, the Logistic function was used to fit. Crack Growth Factor Model (CGFM) was proposed to describe the relationship between the length (and area) of cracks and stress. Fitting curve is $\mathrm{y}=A_{2}+\frac{\left(A_{1}-A_{2}\right)}{\left(1+x / x_{0}\right)^{p}}$, where each parameter in the equation is given practical meaning, the length or area of the cracks in any status is represented with the strain $y, A_{1}$ is used for the length or area of the cracks in the initial state, $A_{2}$ is used for the maximum length that the cracks can reach in the destructive status, Area value, $x_{0}$ is represented with corresponding to the stress value of $A_{2} / 2, p$ is power, as the correlation coefficient. So the original formula becomes $L=$ $L_{2}+\frac{\left(L_{1}-L_{2}\right)}{\left(1+\sigma / \sigma_{0}\right)^{P}}$ or $S=S_{2}+\frac{\left(S_{1}-S_{2}\right)}{\left(1+\sigma / \sigma_{0}\right)^{p}}$. The relationship between the crack lengthstress and crack area-stress curves was fitted under the cyclic load/unload conditions. The fitting parameters were shown in Figs. 5.6 and 5.7. The relevant parameters were shown in Tables 5.6 and 5.7.

Because of the different rock samples, the variation range of cracks length and area is different, that is to say, the S1, S2, L1 and L2 of each sample are different, and because $\sigma_{0}$ is related to the maximum value of length and area, the above

Fig. 5.5 Distribution of Logistic function

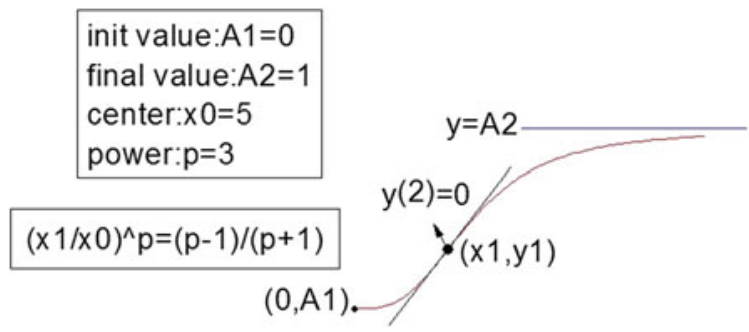




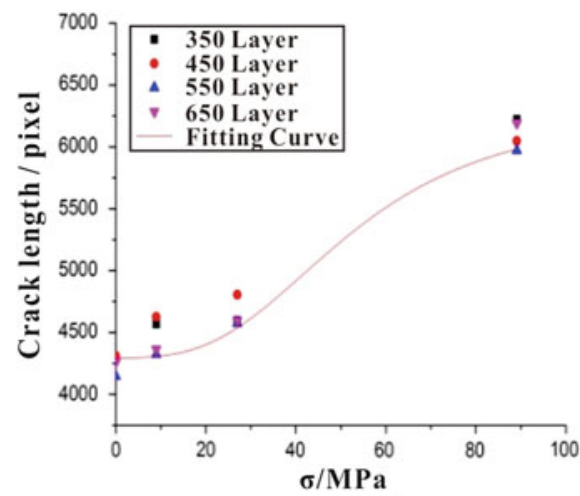

(a) Fitting curve of roof sandstone

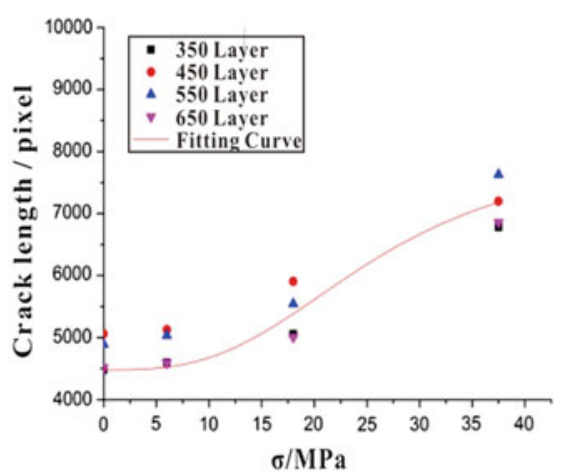

(b) Fitting curve of floor sandstone

Fig. 5.6 Fitting curves of cracks length-stress relation

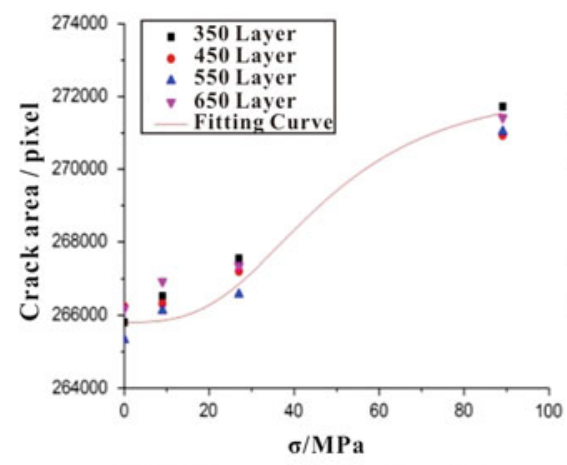

(a) Fitting curve of roof sandstone

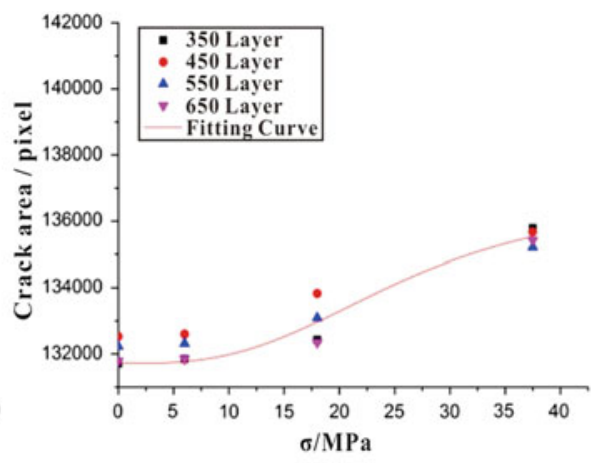

(b) Fitting curve of floor sandstone

Fig. 5.7 Fitting curves of cracks area-stress relation

Table 5.6 Related parameters and equations of fitting curves of crack lengths

\begin{tabular}{l|l|l|l|l|l}
\hline \multirow{2}{*}{ Crack length } & \multicolumn{4}{|l|}{ Related parameters } & Fitting equations \\
\cline { 2 - 5 } & $\mathrm{L}_{1}$ & $\mathrm{~L}_{2}$ & $\sigma_{0}$ & $\mathrm{p}$ & \\
\hline Roof sandstone & 4295.6 & 6330.9 & 52.66 & 3 & $L=6330.9+\frac{(4295.6-6330.9)}{(1+\sigma / 52.66)^{3}}$ \\
\hline Floor sandstone & 4480.8 & 8070.0 & 25.89 & 3 & $L=8070.0+\frac{(4480.8-8070.0)}{(1+\sigma / 25.89)^{3}}$ \\
\hline
\end{tabular}


Table 5.7 Related parameters and equations of fitting curves of crack areas

\begin{tabular}{l|l|l|l|l|l}
\hline \multirow{2}{*}{ Crack areas } & \multicolumn{4}{|l|}{ Related parameters } & Fitting equations \\
\cline { 2 - 6 } & $\mathrm{S}_{1}$ & $\mathrm{~S}_{2}$ & $\sigma_{0}$ & $\mathrm{p}$ & \\
\hline Roof sandstone & 265,810 & 272,380 & 46.95 & 3 & $S=272380+\frac{(265810-272380)}{(1+\sigma / 46.95)^{3}}$ \\
\hline Floor sandstone & 131,710 & 136,830 & 26.15 & 3 & $S=136830+\frac{(131710-136830)}{(1+\sigma / 26.15)^{3}}$ \\
\hline
\end{tabular}

expression is different. However, the evolution of cracks is consistent with the Logistic equation under uniaxial cyclic load/unload. Therefore, the length and area of cracks propagation process can be defined as the parameter $B$, so as to construct the CGFM based on the parameter $B$ :

$$
G=B_{2}+\frac{\left(B_{1}-B_{2}\right)}{\left(1+\sigma / \sigma_{0}\right)^{3}}
$$

where $G$ is growth factor of crack; $B_{1}$ is the initial value of crack parameter; $B_{2}$ is the destruction of the status of cracks can reach the maximum parameter value; $\sigma$ is the stress value; $\sigma_{0}$ is represented with corresponding to the stress value of $B_{2} / 2$; $p$ is the power, the correlation coefficient, CGFM of sandstone $\mathrm{p}=3$.

The model quantitatively describes the propagation and evolution of cracks in the process of sandstone fracture, which provides a theoretical basis for studying the effect of meso-structure change on the macroscopic damage of sandstone. In comparison of roof and floor sandstone, it can be inferred that other rock samples should be coincided with the model. Therefore, the model can be extended to all hard rock. However, there $p$ value of the size will change for different lithology of rock.

$$
G=B_{2}+\frac{\left(B_{1}-B_{2}\right)}{\left(1+\sigma / \sigma_{0}\right)^{\mathrm{p}}}
$$

where $G$ is growth factor of crack; $B_{1}$ is the initial value of crack parameter; $B_{2}$ is the destruction of crack status can reach the maximum parameter value; $\sigma$ is the stress value; $\sigma_{0}$ is represented with corresponding to the stress value of $B_{2} / 2 ; p$ is the power, the correlation coefficient, the selection of $p$ value is related to the lithology of rock sample.

It shows that CGFM is impossible to grow infinitely; the actual situation is that the crack grows to a certain number, and then the rate of crack growth will slow down. Because the objective cause of block effect have a certain impact on the crack growth, and the block effect will continue to increase with the growth of crack, the growth rate of crack is not a constant, it should be reduced. Finally, the crack grows to the maximum value when rock reach to the failure point, then the growth rate is reduced to 0 . In this chapter, the growth factor model of crack is a new attempt to describe growth process of rock crack and a new idea for predicting of rockburst. 


\subsection{Conclusion}

In this chapter, the fractal dimension, length and area of fractures in the whole process of sandstone under uniaxial cyclic load/unload were analyzed, and the quantitative description of CT test was completed, we obtained the following conclusions:

1. Under the cyclic load/unload, the internal cracks of sandstone specimens were gradually sprouted and expanded, which made the complexity of cracks structure increase and the fractal dimension increases. After the stress reached the peak, with the cracking of sandstone specimens, the degree of sample failure continued to increase. However, the shape and structure of cracks tended to be simplified, the complexity was reduced and the fractal dimension was reduced. Therefore, the fractal dimension could be used to quantitatively describe the evolution of cracks propagation in the process of rock failure.

2. The entropy model of rock system was constructed as $S=\mathrm{k} \times \ln \left(D_{0}+A \mathrm{e}^{-\frac{\left(\sigma-\sigma_{\mathrm{c}}\right)}{2 \times \omega}}\right)$. Under uniaxial cyclic load/unload, the fractal dimension change of cracks was combined with the evolution of quantitatively described system entropy. It was proved that the dimensionality reduction, entropy reduction and order were unified in the process of rock failure. The dynamic characteristics of rock system, which was from disorder to order, could be described by the process of dimension reduction and entropy reduction.

3. The evolution formula of CT number for sandstone under uniaxial cyclic load/ unload could be expressed by the equation after the corresponding transformation. Therefore, the evolution process of density of sandstone could be described by one-dimensional chaotic mapping Logistic equation under uniaxial cyclic load/ unload, and the evolution process of sandstone crack had chaotic characteristics.

4. In this chapter, CGFM was established with the equation $G=B_{2}+\frac{\left(B_{1}-B_{2}\right)}{\left(1+\sigma / \sigma_{0}\right)^{3}}$, which quantitatively described the evolution of crack propagation in the process of rock failure. When the cracks growed to a certain number, the growth rate of cracks increased with the blocking effect, then the rock was failure, the cracks growed to the maximum, and the crack growth rate became zero at this time.

\section{References}

Bobet A (2000) The initiation of secondary cracks in compression. Eng Fract Mech 66(2):187-219

Faloutsos C, Gaede V (1996) Analysis of n-dimensional quadtrees using the Hausdorff fractal dimension. Comput Sci Dep 548

Foroutan-pour K, Dutilleul P, Smith DL (1999) Advances in the implementation of the box-counting method of fractal dimension estimation. Appl Math Comput 105(2):195-210

Goldberger AL, Amaral LAN, Hausdorff JM et al (2002) Fractal dynamics in physiology: alterations with disease and aging. Proc Natl Acad Sci 99(suppl 1):2466-2472 
Heping X (1989) Studies on fractal models of the microfracture of marble. Kexue tongbao (English edition) 34(15):1292-1296

Hoxha D, Lespinasse M, Sausse J et al (2005) A microstructural study of natural and experimentally induced cracks in a granodiorite. Tectonophysics 395(1):99-112

Hudson JA, Priest SD (1979) Discontinuities and rock mass geometry. Int J Rock Mech Min Sci 16(6): 339-362

Li J, Du Q, Sun C (2009) An improved box-counting method for image fractal dimension estimation. Pattern Recogn 42(11):2460-2469

Lin P, Wong RHC, Chau KT et al (2000) Multi-crack coalescence in rock-like material under uniaxial and biaxial loading. Key Eng Mater 183:809-814

Mandelbrot BB (1977) Fractals. Wiley, New York

Mandelbrot BB (1999) Fractal object: the shape, the opportunities and dimension. World Publishing Corporation, Beijing

Mandelbrot BB, Pignoni R (1983) The fractal geometry of nature. WH freeman, New York

Nolen-Hoeksema RC, Gordon RB (1987) Optical detection of crack patterns in the opening-mode fracture of marble. Int J Rock Mech Min Sci 24(2):135-144

Priest SD, Hudson JA (1976) Discontinuity spacings in rock. Int J Rock Mech Min Sci 13(5):135148

Reyes O, Einstein HH (1991) Failure mechanisms of fractured rock-a fracture coalescence model. Int Soc Rock Mech 10(1):333-340

Shen B, Barton N (1997) The disturbed zone around tunnels in jointed rock masses. Int J Rock Mech Min Sci 34(1):117-125

Shen B, Stephansson O, Einstein HH et al (1995) Coalescence of fractures under shear stresses in experiments. J Geophys Res 100(6):5975-5990

Wong RHC, Chau KT, Tang CA et al (2001) Analysis of crack coalescence in rock-like materials containing three flaws - part I: experimental approach. Int J Rock Mech Min Sci 38(7):909924

Wong TF (1982) Micromechanics of faulting in Westerly granite. Int J Rock Mech Min Sci 19 (2):49-64

Wong TF, Wong RHC, Jiao MR et al (2004) Micromechanics and rock failure process analysis. Key Eng Mater 261(263): 1

Xie Heping, Chen Zhida, (1988) Fractal geometry and fracture of rock. Acta Mechanica Sinica 4 (3):255-264

Open Access This chapter is licensed under the terms of the Creative Commons Attribution-NonCommercial 4.0 International License (http://creativecommons.org/licenses/by-nc/ 4.0/), which permits any noncommercial use, sharing, adaptation, distribution and reproduction in any medium or format, as long as you give appropriate credit to the original author(s) and the source, provide a link to the Creative Commons license and indicate if changes were made.

The images or other third party material in this book are included in the book's Creative Commons license, unless indicated otherwise in a credit line to the material. If material is not included in the book's Creative Commons license and your intended use is not permitted by statutory regulation or exceeds the permitted use, you will need to obtain permission directly from the copyright holder. 